\title{
The Stäckel theorem in the Lagrangian formalism and the use of local times
}

\author{
G. F. Torres del Castillo \\ Instituto de Ciencias Universidad Autónoma de Puebla, \\ 72570 Puebla, Pue., México.
}

Received 23 December 2020; accepted 21 January 2021

\begin{abstract}
We show that the conditions for the separability of the Hamilton-Jacobi equation given by the Stäckel theorem imply that, using the elementary Lagrangian formalism, one can find $n$ functionally independent constants of motion, where $n$ is the number of degrees of freedom. We also show that this result can be linked to the fact that the Lagrangian for a system of this class is related to the sum of $n$ one-dimensional Lagrangians, if one makes use of multiple local times.
\end{abstract}

Keywords: Stäckel's theorem; Lagrangian formulation; fictitious time.

Mostramos que las condiciones para la separabilidad de la ecuación de Hamilton-Jacobi dadas por el teorema de Stäckel implican que, usando el formalismo Lagrangiano elemental, se pueden hallar $n$ constantes de movimiento funcionalmente independientes, donde $n$ es el número de grados de libertad. Mostramos también que este resultado puede ligarse al hecho de que la lagrangiana para un sistema de esta clase está relacionada con la suma de $n$ lagrangianas unidimensionales, si se utilizan múltiples tiempos locales.

Descriptores: Teorema de Stäckel; formulación lagrangiana; tiempo ficticio.

PACS: 45.20.Jj; 02.30.Hq

DOI: https://doi.org/10.31349/RevMexFis.67.447

\section{Introduction}

The standard method for solving the Hamilton-Jacobi equation is that of separation of variables, and, therefore, it is interesting to know the conditions for the existence of complete separable solutions for this equation. A partial answer to this question is provided by the Stäckel theorem (see, e.g., Refs. [1-4]), which gives necessary and sufficient conditions for the existence of complete separable solutions of the Hamilton-Jacobi equation for certain orthogonal systems (here orthogonal means that the kinetic energy has the form $(1 / 2) \sum_{i=1}^{n} \dot{q}_{i}{ }^{2} / c_{i}$, where the $c_{i}$ are functions of the generalized coordinates only).

A more restricted class of systems than those included in the Stäckel theorem are the Liouville systems (see, e.g., Refs. [1, 2, 4]). In a recent paper [5], it is shown that the form of the Lagrangian of the Liouville systems can be obtained starting from a sum of one-dimensional Lagrangians expressed in terms of a fictitious (or local) time, which replaces the real time. The aim of this paper is to show that, in a similar way, the conditions of the Stäckel theorem are related to several fictitious times.

In Sec. 2, the Stäckel theorem is enunciated, following Refs. [1,2], and we find a general expression for $n$ constants of motion possessed by any Lagrangian satisfying the conditions of the Stäckel theorem. These constants of motion are essentially the separation constants appearing in the solution of the Hamilton-Jacobi equation. In Sec. 3, we show that the motion of a system satisfying the conditions of the Stäckel theorem can be viewed as the composition of $n$ independent one-dimensional systems by introducing a possibly different fictitious (or local) time for each coordinate $q_{i}$.

\section{The Stäckel theorem}

The Stäckel theorem is applicable to a system described by a Lagrangian of the form

$$
L=\sum_{i=1}^{n}\left(\frac{\dot{q}_{i}^{2}}{2 c_{i}}-c_{i} w_{i}\right),
$$

where the functions $c_{i}$ may depend on the $n$ generalized coordinates $q_{1}, q_{2}, \ldots, q_{n}$ only, and $w_{i}$ is a function of the single variable $q_{i}$ (throughout this paper, the summation convention is not employed; all the sums are explicitly indicated). According to this theorem, the Hamilton-Jacobi equation corresponding to (1) admits separable solutions in this coordinate system if and only if there exists a non-singular $n \times n$ matrix $\left(u_{i j}\right)$, where $u_{i j}$ is a function of $q_{i}$ only (that is, the entries in the first row of $\left(u_{i j}\right)$ are functions of $q_{1}$ only, and so on) such that

$$
\sum_{i=1}^{n} c_{i} u_{i k}=\delta_{1 k}
$$

This last condition implies that if $\left(\tilde{u}_{i j}\right)$ is the inverse of the matrix $\left(u_{i j}\right)$, then

$$
\tilde{u}_{1 i}=c_{i} .
$$

For example, for a charged particle in the field of a point dipole, in terms of the spherical coordinates $(r, \theta, \phi)$, the natural Lagrangian is

$$
L=\frac{m}{2}\left(\dot{r}^{2}+r^{2} \dot{\theta}^{2}+r^{2} \sin ^{2} \theta \dot{\phi}^{2}\right)-\frac{k \cos \theta}{r^{2}},
$$


where $k$ is a constant. This Lagrangian has the form (1) with

$$
c_{1}=\frac{1}{m}, \quad c_{2}=\frac{1}{m r^{2}}, \quad c_{3}=\frac{1}{m r^{2} \sin ^{2} \theta},
$$

if we take $\left(q_{1}, q_{2}, q_{3}\right)=(r, \theta, \phi)$, and we can choose (though this is not the only possibility)

$$
w_{1}(r)=0, \quad w_{2}(\theta)=m k \cos \theta, \quad w_{3}(\phi)=0 .
$$

Then, a matrix $\left(u_{i j}\right)$ that satisfies condition (2) is

$$
\left(\begin{array}{ccc}
m & 0 & -1 / r^{2} \\
0 & 1 / \sin ^{2} \theta & 1 \\
0 & -1 & 0
\end{array}\right) .
$$

(Note that the first row of this matrix is a function of $r$ only, the second row is a function of $\theta$ only, and the third row is a function of $\phi$ only. Contrariwise to the claim in Ref. [6], it is a straightforward matter to verify if the conditions of the Stäckel theorem are satisfied; see also the example at the end of this section).

Thus, the Stäckel theorem guarantees that the HamiltonJacobi equation for this problem, in these coordinates, admits complete separable solutions (see, e.g., Ref. [7]). However, instead of exhibiting this separability, we shall consider the more elementary problem of solving the Lagrange equations: Substituting the Lagrangian (4) into the Lagrange equations we obtain

$$
\begin{aligned}
& 0=\frac{\mathrm{d}}{\mathrm{d} t}(m \dot{r})-m r \dot{\theta}^{2}-m r \sin ^{2} \theta \dot{\phi}^{2}-\frac{2 k \cos \theta}{r^{3}}, \\
& 0=\frac{\mathrm{d}}{\mathrm{d} t}\left(m r^{2} \dot{\theta}\right)-m r^{2} \sin \theta \cos \theta \dot{\phi}^{2}-\frac{k \sin \theta}{r^{2}}, \\
& 0=\frac{\mathrm{d}}{\mathrm{d} t}\left(m r^{2} \sin ^{2} \theta \dot{\phi}\right) .
\end{aligned}
$$

The last of these equations means that the quantity inside the parentheses is a constant of motion:

$$
m r^{2} \sin ^{2} \theta \dot{\phi}=l_{3},
$$

where $l_{3}$ is a constant (that represents the $z$-component of the angular momentum and the momentum conjugate to $\phi$ ). Making use of this relation in order to eliminate $\dot{\phi}$ from Eq. (7), we obtain

$$
0=\frac{\mathrm{d}}{\mathrm{d} t}\left(m r^{2} \dot{\theta}\right)-\frac{l_{3}^{2} \cos \theta}{m r^{2} \sin ^{3} \theta}-\frac{k \sin \theta}{r^{2}} .
$$

Multiplying this equation by $2 m r^{2} \dot{\theta}$, we find that

$$
\begin{aligned}
0 & =2 m r^{2} \dot{\theta} \frac{\mathrm{d}}{\mathrm{d} t}\left(m r^{2} \dot{\theta}\right)-\frac{2 l_{3}^{2} \cos \theta \dot{\theta}}{\sin ^{3} \theta}-2 m k \sin \theta \dot{\theta} \\
& =\frac{\mathrm{d}}{\mathrm{d} t}\left(\left[m r^{2} \dot{\theta}\right]^{2}+\frac{l_{3}^{2}}{\sin ^{2} \theta}+2 m k \cos \theta\right),
\end{aligned}
$$

which yields a second constant of motion:

$$
\left(m r^{2} \dot{\theta}\right)^{2}+\frac{l_{3}^{2}}{\sin ^{2} \theta}+2 m k \cos \theta=M
$$

(when $k=0, M$ reduces to the square of the angular momentum).

With the aid of Eqs. (9) and (11) now we can eliminate $\dot{\phi}$ and $\dot{\theta}$ from Eq. (6); the result is

$$
0=\frac{\mathrm{d}}{\mathrm{d} t}(m \dot{r})-\frac{M}{m r^{3}}
$$

(not only $\dot{\phi}$ and $\dot{\theta}$ were eliminated, $\phi$ and $\theta$ also disappeared) hence, multiplying by $\dot{r}$,

$$
0=\dot{r} \frac{\mathrm{d}}{\mathrm{d} t}(m \dot{r})-\frac{M \dot{r}}{m r^{3}}=\frac{\mathrm{d}}{\mathrm{d} t}\left(\frac{m}{2} \dot{r}^{2}+\frac{M}{2 m r^{2}}\right)
$$

This means that the expression inside the parentheses is also a constant of motion, which turns out to be the total energy [see Eqs. (4), (9) and (11)]:

$$
\frac{m}{2} \dot{r}^{2}+\frac{M}{2 m r^{2}}=E
$$

where $E$ is a third constant of motion (whose existence follows directly from the fact that the Lagrangian does not depend explicitly of the time).

Thus, despite the mixture of the coordinates in Eqs. (6)(8), miraculously, one obtains three first-order differential equations or, equivalently, three first integrals [Eqs. (9), (11), and (13)]. As we shall show now, this is a consequence of the fulfillment of the conditions of the Stäckel theorem, and a similar reduction can be obtained for any Lagrangian of the form (1), if the conditions of the Stäckel theorem are satisfied.

Proposition. For a Lagrangian of the form (1), satisfying the conditions of the Stäckel theorem, the $n$ quantities

$$
\alpha_{i} \equiv \sum_{j=1}^{n} \tilde{u}_{i j}\left(\frac{1}{2}\left[\frac{\dot{q}_{j}}{c_{j}}\right]^{2}+w_{j}\right)
$$

are constants of motion. In particular, $\alpha_{1}$ is the Jacobi integral $\sum_{i=1}^{n}\left(\dot{q}_{i}^{2} / 2 c_{i}+c_{i} w_{i}\right)$.

Proof. Making use of the definition (14), one finds that the time derivative of $\alpha_{i}$ is

$$
\begin{aligned}
\frac{\mathrm{d} \alpha_{i}}{\mathrm{~d} t} & =\sum_{j=1}^{n}\left(\tilde{u}_{i j}\left[\frac{\dot{q}_{j}}{c_{j}} \frac{\mathrm{d}}{\mathrm{d} t}\left\{\frac{\dot{q}_{j}}{c_{j}}\right\}+\frac{\mathrm{d} w_{j}}{\mathrm{~d} q_{j}} \dot{q}_{j}\right]\right. \\
& \left.-\left[\frac{1}{2}\left\{\frac{\dot{q}_{j}}{c_{j}}\right\}^{2}+w_{j}\right] \sum_{k, l=1}^{n} \tilde{u}_{i k} \frac{\mathrm{d} u_{k l}}{\mathrm{~d} q_{k}} \dot{q}_{k} \tilde{u}_{l j}\right),
\end{aligned}
$$

where we have made use of the fact that $w_{j}$ and $u_{j k}$ are functions of the single variable $q_{j}$ and that, for any non-singular matrix $A, \mathrm{~d} A^{-1}=-A^{-1}(\mathrm{~d} A) A^{-1}$. On the other hand, substitution of the Lagrangian (1) into the Lagrange equations 
gives, for $j=1,2, \ldots, n$,

$$
\begin{aligned}
0=\frac{\mathrm{d}}{\mathrm{d} t}\left(\frac{\dot{q}_{j}}{c_{j}}\right) & +\frac{1}{2} \sum_{i=1}^{n} \frac{1}{c_{i}^{2}} \frac{\partial c_{i}}{\partial q_{j}}\left(\dot{q}_{i}\right)^{2}+\sum_{i=1}^{n} \frac{\partial c_{i}}{\partial q_{j}} w_{i}+c_{j} \frac{\mathrm{d} w_{j}}{\mathrm{~d} q_{j}} \\
& =c_{j}\left(\frac{1}{c_{j}} \frac{\mathrm{d}}{\mathrm{d} t}\left[\frac{\dot{q}_{j}}{c_{j}}\right]+\frac{\mathrm{d} w_{j}}{\mathrm{~d} q_{j}}\right) \\
& +\sum_{i=1}^{n} \frac{\partial c_{i}}{\partial q_{j}}\left(\frac{1}{2}\left[\frac{\dot{q}_{i}}{c_{i}}\right]^{2}+w_{i}\right) .
\end{aligned}
$$

Taking the partial derivative with respect to $q_{j}$ on both sides of Eq. (2), one obtains

$$
0=c_{j} \frac{\mathrm{d} u_{j k}}{\mathrm{~d} q_{j}}+\sum_{i=1}^{n} \frac{\partial c_{i}}{\partial q_{j}} u_{i k}
$$

which implies that

$$
\sum_{k, l=1}^{n} \tilde{u}_{i k} \frac{\mathrm{d} u_{k l}}{\mathrm{~d} q_{k}} \dot{q}_{k} \tilde{u}_{l j}=-\sum_{k=1}^{n} \tilde{u}_{i k} \frac{1}{c_{k}} \frac{\partial c_{j}}{\partial q_{k}} \dot{q}_{k} .
$$

Substituting Eqs. (16) and (18) into (15) one finds that, in effect, $\mathrm{d} \alpha_{i} / \mathrm{d} t=0$.

Finally, with the aid of Eqs. (14) and (3) we see that

$$
\alpha_{1}=\sum_{j=1}^{n} c_{j}\left(\frac{1}{2}\left[\frac{\dot{q}_{j}}{c_{j}}\right]^{2}+w_{j}\right),
$$

which is the Jacobi integral corresponding to the Lagrangian (1).

Thus, we have the expression for $n$ functionally independent constants of motion, which reduce the equations of motion to quadratures. In fact, Eqs. (14) can be inverted to give

$$
\frac{1}{2}\left(\frac{\dot{q}_{j}}{c_{j}}\right)^{2}+w_{j}=\sum_{k=1}^{n} u_{j k} \alpha_{k} .
$$

(Remember that $w_{j}$ and $u_{j k}$ are functions of the single variable $q_{j}$.)

Going back to the case of the Lagrangian (4), one readily finds that the inverse of the matrix $(5)$ is given by

$$
\left(\tilde{u}_{i j}\right)=\left(\begin{array}{ccc}
1 / m & 1 / m r^{2} & 1 / m r^{2} \sin ^{2} \theta \\
0 & 0 & -1 \\
0 & 1 & 1 / \sin ^{2} \theta
\end{array}\right)
$$

[cf. Eq. (3)] and that the constants of motion (14) are related to the constants of motion previously obtained, (9), (11), and (13), by $\alpha_{1}=E, \alpha_{2}=-(1 / 2) l_{3}^{2}$, and $\alpha_{3}=(1 / 2) M$.

Another illustrative example is given by the Lagrangian

$$
\begin{aligned}
L & =\frac{m}{2}\left[\left(u^{2}+v^{2}\right)\left(\dot{u}^{2}+\dot{v}^{2}\right)+u^{2} v^{2} \dot{\phi}^{2}\right] \\
& +\frac{2 k}{u^{2}+v^{2}}+\frac{\gamma}{2}\left(u^{2}-v^{2}\right),
\end{aligned}
$$

where $(u, v, \phi)$ are parabolic coordinates, which are related to the Cartesian ones by $x=u v \cos \phi, y=u v \sin \phi$, $z=(1 / 2)\left(u^{2}-v^{2}\right)$, and $k, \gamma$ are constants. (This Lagrangian corresponds to a charged particle of mass $m$, in the field of a point charge placed at the origin, and a uniform field in the $z$-direction.) Clearly, in this example,

$$
c_{1}=\frac{1}{m\left(u^{2}+v^{2}\right)}=c_{2}, \quad c_{3}=\frac{1}{m u^{2} v^{2}},
$$

and the one-variable functions $w_{j}$ can be taken as

$$
\begin{aligned}
& w_{1}(u)=-m k-\frac{m \gamma}{2} u^{4}, \quad w_{2}(v)=-m k+\frac{m \gamma}{2} v^{4}, \\
& w_{3}(\phi)=0 .
\end{aligned}
$$

Equation (2) is satisfied by the matrix

$$
\left(u_{i j}\right)=\left(\begin{array}{ccc}
m u^{2} & 1 / u^{2} & 1 \\
m v^{2} & 1 / v^{2} & -1 \\
0 & -1 & 0
\end{array}\right)
$$

and, therefore, the conditions of the Stäckel theorem are met.

The matrix $\left(\tilde{u}_{i j}\right)$ is

$$
\frac{1}{m\left(u^{2}+v^{2}\right)}\left(\begin{array}{ccc}
1 & 1 & 1 / u^{2}+1 / v^{2} \\
0 & 0 & -m\left(u^{2}+v^{2}\right) \\
m v^{2} & -m u^{2} & m\left(v^{2} / u^{2}-u^{2} / v^{2}\right)
\end{array}\right),
$$

and, from Eq. (14), we have immediately the two constants of motion (apart from the total energy $\alpha_{1}$ ),

$$
\begin{aligned}
\alpha_{2} & =-\frac{p_{\phi}{ }^{2}}{2} \\
\alpha_{3} & =\frac{m^{2}}{2}\left[\left(u^{2}+v^{2}\right)\left(v^{2} \dot{u}^{2}-u^{2} \dot{v}^{2}\right)+u^{2} v^{2}\left(v^{2}-u^{2}\right) \dot{\phi}^{2}\right] \\
& -\frac{m \gamma}{2} u^{2} v^{2}+m k \frac{u^{2}-v^{2}}{u^{2}+v^{2}},
\end{aligned}
$$

where $p_{\phi}$ is the momentum conjugate to $\phi$ (which is conserved as a consequence of the fact that $\phi$ is an ignorable coordinate). The constant of motion $\alpha_{3}$ is not related to obvious symmetries and, when $\gamma$ is set equal to zero, reduces to minus the $z$-component of the Laplace-Runge-Lenz vector. (Of course, as is well known, any constant of motion corresponds to a one-parameter group of symmetries of the Hamiltonian in the phase space; however, $\alpha_{3}$ is not related to a variational symmetry of the Lagrangian; in fact, the Laplace-RungeLenz vector is the standard example of a constant of motion associated with a hidden or accidental symmetry.)

In this case, Eqs. (19) amount to

$$
\begin{aligned}
\frac{1}{2}\left(m\left(u^{2}+v^{2}\right) \dot{u}\right)^{2}-m k-\frac{m \gamma}{2} u^{4} & =\alpha_{1} m u^{2}+\frac{\alpha_{2}}{u^{2}}+\alpha_{3} \\
\frac{1}{2}\left(m\left(u^{2}+v^{2}\right) \dot{v}\right)^{2}-m k+\frac{m \gamma}{2} v^{4} & =\alpha_{1} m v^{2}+\frac{\alpha_{2}}{v^{2}}-\alpha_{3} \\
\frac{1}{2}\left(m u^{2} v^{2} \dot{\phi}\right)^{2} & =-\alpha_{2}
\end{aligned}
$$

[cf. Eqs. (9), (11) and (13)]. These equations are equivalent to those obtained using the Hamilton-Jacobi equation. 


\section{Local times}

Even though Eqs. (9), (11) and (12) contain the time derivative of a single coordinate, Eqs. (9) and (11) still contain mixtures of the coordinates. This fact can be conveniently hidden by introducing local (or fictitious) times, $\tau_{i}$, defined by

$$
\mathrm{d} \tau_{i} \equiv c_{i} \mathrm{~d} t
$$

(see, e.g., Refs. [8,5], and the references cited therein).

Considering again the example provided by the Lagrangian (4), we have the three local times

$$
\mathrm{d} \tau_{1}=\frac{1}{m} \mathrm{~d} t, \quad \mathrm{~d} \tau_{2}=\frac{1}{m r^{2}} \mathrm{~d} t, \quad \mathrm{~d} \tau_{3}=\frac{1}{m r^{2} \sin ^{2} \theta} \mathrm{d} t .
$$

We see that only $\mathrm{d} \tau_{1}$ is an exact differential, and $\tau_{1}$ is, up to a constant factor, the real time. For instance, in terms of $\tau_{2}$, Eqs. (10) and (11) take the form

$$
\frac{\mathrm{d}^{2} \theta}{\mathrm{d} \tau_{2}^{2}}-\frac{l_{3}^{2} \cos \theta}{\sin ^{3} \theta}-m k \sin \theta=0
$$

and

$$
\left(\frac{\mathrm{d} \theta}{\mathrm{d} \tau_{2}}\right)^{2}+\frac{l_{3}^{2}}{\sin ^{2} \theta}+2 m k \cos \theta=M,
$$

respectively, which determine $\theta$ as a function of $\tau_{2}$ alone. Furthermore, one can readily verify that Eq. (22) is the Lagrange equation for the one-dimensional Lagrangian

$$
\begin{aligned}
L_{2}\left(\theta, \mathrm{d} \theta / \mathrm{d} \tau_{2}, \tau_{2}\right) & \equiv \frac{1}{2}\left(\frac{\mathrm{d} \theta}{\mathrm{d} \tau_{2}}\right)^{2}-\frac{l_{3}^{2}}{2 \sin ^{2} \theta} \\
& -m k \cos \theta+\text { const. },
\end{aligned}
$$

treating $l_{3}$ as a constant, and that the left-hand side of Eq. (23) is, essentially, the Jacobi integral corresponding to $L_{2}$, multiplied by 2 .

Again, this is not an exceptional behavior; making use of Eqs. (21), (14) and (17) we see that the Lagrange equations (16) are equivalent to

$$
0=\frac{\mathrm{d}^{2} q_{j}}{\mathrm{~d} \tau_{j}^{2}}+\frac{\mathrm{d} w_{j}}{\mathrm{~d} q_{j}}-\sum_{k=1}^{n} \frac{\mathrm{d} u_{j k}}{\mathrm{~d} q_{j}} \alpha_{k}
$$

$(j=1,2, \ldots, n)$, which, for each value of $j$, is the Lagrange equation for the one-dimensional Lagrangian

$$
L_{j}\left(q_{j}, \mathrm{~d} q_{j} / \mathrm{d} \tau_{j}, \tau_{j}\right) \equiv \frac{1}{2}\left(\frac{\mathrm{d} q_{j}}{\mathrm{~d} \tau_{j}}\right)^{2}-w_{j}+\sum_{k=1}^{n} u_{j k} \alpha_{k},
$$

provided that the $\alpha_{k}$ are treated as constants. (Recall that $w_{j}$ and $u_{j k}$ are functions of the single variable $q_{j}$.) Making use of Eqs. (26), (21), (2), and (1), one finds that the one-dimensional Lagrangians (26) are related to the original Lagrangian (1) by

$$
\sum_{j=1}^{n} L_{j} \mathrm{~d} \tau_{j}=(L+E) \mathrm{d} t
$$

[cf. Ref. [5], Eq. (21)]. The combination $(L+E) \mathrm{d} t$ appears in the principle of least action (instead of $L \mathrm{~d} t$, considered in Hamilton's principle), which is applicable when $E$ is conserved on the actual and the varied paths (see, e.g., Refs. $[9,10])$.

Since the Lagrangian $L_{j}$ does not depend on $\tau_{j}$, the corresponding Jacobi integral is a constant of motion. Making use of the definitions (26), (21), and (14), one finds that these constants of motion are identically equal to zero. (By contrast, in the case of a Liouville system, where a single local time is enough, the Jacobi integrals associated with the onedimensional Lagrangians lead to $n-1$ nontrivial constants of motion [5]).

\section{Concluding remarks}

With the only exception of $\alpha_{1}$, which is related to the timeindependence of the Lagrangian (1), the other $n-1$ constants of motion, $\alpha_{2}, \ldots, \alpha_{n}$, need not be associated with ignorable coordinates.

As we have shown, the Stäckel theorem, which is related to the separability of the Hamilton-Jacobi equation, turns out to be directly relevant in the Lagrangian formalism. This fact must not be surprising since, in both formalisms, one is dealing with the same problem. In order to have an analog of the Stäckel theorem in the Lagrangian formalism, without reference to the Hamilton-Jacobi equation, it would be necessary to have an analog of the concept of separability.

\section{Acknowledgement}

The author wishes to thank the referee for stimulating comments.
1. L.A. Pars, A Treatise on Analytical Dynamics (Wiley, New York, 1965; reprinted by Ox Bow Press, 1979), Sec. 18.2. doi.org/10.2307/3612016

2. D.T. Greenwood, Classical Dynamics (Prentice-Hall, Englewood Cliffs, New Jersey, 1977; reprinted by Dover, 1997), Sec. 5-3.
3. H. Goldstein, Classical Mechanics, 2nd ed. (Addison-Wesley, Reading, MA, 1980), Sec. 10-4 and Appendix D.

4. A.M. Perelomov, Integrable Systems of Classical Mechanics and Lie Algebras, Vol. I (Birkhäuser, Basel, 1990), Sect. 2.3. doi.org/10.1007/978-3-0348-9257-5 
5. G.F. Torres del Castillo, The use of fictitious time in Lagrangian mechanics, Rev. Mex. Fís. E (to appear).

6. D.T. Greenwood, op. cit., p. 208.

7. G.F. Torres del Castillo, An Introduction to Hamiltonian Me- chanics (Springer, Cham, 2018), Example 6.5.doi.org/10. 1007/978-3-319-95225-3

8. L.A. Pars, op. cit., Sec. 18.3.

9. L.A. Pars, op. cit., Sec. 27.2.

10. H. Goldstein, op. cit., Sec. 8-6. 